\title{
Clinical observation of warming acupuncture and moxibustion at the temples combined with Deanxit in the treatment of tension headache with anxiety and depression: a retrospective study
}

\author{
Runa $\mathrm{A}^{1 \#}$, Qinglin $\mathrm{Bao}^{2 \#}$, Yin Chaoketu Sai ${ }^{2}, \mathrm{Muqile}^{\mathrm{Te}}{ }^{2}$, Rilebagen $\mathrm{Hu}^{1}$, Rentuya $\mathrm{Sa}^{3}$, Rigenjiya $\mathrm{Mu}^{1}$, \\ Agula $\mathrm{Bo}^{4}$ \\ ${ }^{1}$ Mongolian Medicine School, Inner Mongolia Medical University, Hohhot, China; ${ }^{2}$ Department of Five Therapy for Encephalopathy, Inner \\ Mongolia International Mongolian Medicine Hospital, Hohhot, China; ${ }^{3}$ Department of Mongolian Medicine, Affiliated Hospital of Inner Mongolia \\ Medical University, Hohhot, China; ${ }^{4}$ Baotou Medical College, Baotou, China \\ Contributions: (I) Conception and design: All authors; (II) Administrative support: All authors; (III) Provision of study materials or patients: All \\ authors; (IV) Collection and assembly of data: All authors; (V) Data analysis and interpretation: All authors; (VI) Manuscript writing: All authors; (VII) \\ Final approval of manuscript: All authors. \\ \#These authors contributed equally to this work. \\ Correspondence to: Agula Bo. Baotou Medical College, 31 Jianshe Road, Donghe District, Baotou 014040, China. Email: agula14526@163.com.
}

\begin{abstract}
Background: Tension-type headache (TTH), also called muscle contraction headache or neurological headache, is mainly characterized by chronic and persistent bilateral headache in the neck and a severe sense of restraint in the head. This study aims to analyze the effect of warming acupuncture and moxibustion at temples combined with Deanxit on tension headache.

Methods: A total of 252 patients with anxiety and tension headache were randomly divided into routine group and study group. The study group was treated with Dailixin on the basis of warm acupuncture and moxibustion. The headache score, pericranial muscle tenderness score, therapeutic effect, tension headache attack times and duration, HAMD and HAMA scores were analyzed before and after treatment.

Results: The effective cure rate of the study group was significantly higher than that of the routine group. The pericranial muscle tenderness scores of the study group were significantly lower than those of the routine group. Furthermore, the headache degree score, number of attacks, and duration of the study group after treatment were significantly lower than those of the routine group. And the HAMD and HAMA scores in the study group was significantly lower than those in the routine group.

Conclusions: The use of warming acupuncture and moxibustion at temples combined with Deanxit in the treatment of tension headache significantly reduces the number and duration of headache attacks and decreases the degree of headache.
\end{abstract}

Keywords: Warm acupuncture; tension headache; Deanxin; clinical effect

Submitted Mar 16, 2021. Accepted for publication May 21, 2021.

doi: $10.21037 /$ apm-21-925

View this article at: http://dx.doi.org/10.21037/apm-21-925

\section{Introduction}

Tension-type headache (TTH), also called muscle contraction headache or neurological headache $(1,2)$, is mainly characterized by chronic and persistent bilateral headache in the neck and a severe sense of restraint in the head, often accompanied by stress and tension (3). Symptoms of TTC are usually accompanied by dizziness, insomnia, anxiety, depression, or gastrointestinal discomfort. When accompanied by anxiety or depression, TTH has a more serious impact on people's health (4). 
In addition, chronic improper positioning of the head, neck, shoulder, and back muscles (5) leads to muscular cramps and contractions of the neck or head and associated pain, which can be related to surgery, traumatic infection, cervical spondylosis, and other pathological stimuli $(6,7)$. The incidence of TTH is increasing in tandem with the increasing pressures of life in modern society and acceleration of the pace of work; the likelihood of an individual experiencing TTH during their lifetime has increased to about $69 \%$ (8). Mental factors are the most common causes of TTH, including anxiety, tension, fatigue, and so on. Due to the low level of awareness of this disease, patients often exist a state of untreated tension for long periods, engendering headaches that cannot be completely cured $(9,10)$. The risk of depression in patients with tension-type headache is more than 3 times higher than that in normal people, while the probability of tension-type headache in patients with depression is more than 3 times higher than that in non-depressive people. It is suggested that tension-type headache may lead to the occurrence of anxiety and depression. There is a two-way relationship between tension-type headache and anxiety-depression disorder that influence and promote each other. According to foreign literature, the incidence of depression in patients with tension-type headache is $3.8-57 \%$. Tension-type headache, anxiety and depression influence each other and cause and effect each other, seriously affecting the quality of life of patients. Western medicine mainly includes muscle relaxants, such as eperisone, pain relieving drugs, such as phenolic caffeine tablets, acetaminophen, etc., and drugs to improve depression and anxiety, such as amitriptyline, Dailexin, sertraline and so on. It is well known that traditional Chinese medicine (TCM), as well as acupuncture and moxibustion, have good curative effects and few side effects (11); this study adopted the method of warming acupuncture and moxibustion combined with Dailixin in the treatment of TTH of anxiety and depression. The related therapeutic effects of different measures, severity of headache, number of attacks, and duration were compared, analyzed, and reported.

We present the following article in accordance with the STROBE reporting checklist (available at http://dx.doi. org/10.21037/apm-21-925).

\section{Methods}

\section{General information}

A total of 252 patients with anxiety and TTH treated in our hospital from March 2016 to February 2019 were randomly divided into a study group $(\mathrm{n}=126)$ and routine group $(\mathrm{n}=126)$. The selected patients were experiencing TTH attack at the time of the study. The study group included 61 males and 65 females, aged $18-70$ years $(26.9 \pm 15.9$ years), and the course of disease was $3.2 \pm 4.9$ years; the routine group included 70 males and 56 females, aged $20-75$ years old $(29.5 \pm 19.4$ years $)$, and the average course of disease was $3.9 \pm 5.0$ ) years. There was no significant difference in gender, age, and course of disease between the two groups $(\mathrm{P}>0.05)$.

\section{Experimental method}

The diagnostic criteria of TTH can be judged according to the classification and diagnostic criteria of headache, cranial neuralgia, and facial pain formulated by the International Headache Association in 1988. In the classification of tension headache, TIH is divided into two types, including episodic tension headache (ETTH) and chronic tension headache (CTTH). The difference between ETTH and CTTH is that those with ETTH experience headaches for 30 minutes at least 10 times a month, while those with CTTH have at least 15 headaches a month. This study explored the treatment of TTH via two different approaches.

The conventional group received warm acupuncture therapy, predominantly around the temple area. With the patient in the supine position and facing the doctor, the area to be needled was located and routinely disinfected. A disposable aseptic acupuncture needle with a length of 25-40 mm was inserted into the Fengchi acupoint (GB20), penetrating about 1.0 inch toward the tip of the nose, slightly rotated to induce numb feeling elicited locally, and manipulated upward to the posterior occipital bone. To achieve the depth necessary for acupuncture at the temple, the straight needle method was used to insert the needle (length 1.5 inches), perpendicularly penetrate, slightly lift and rotate, then flatten and release the needle after obtaining qi, so that the needle is retained in situ for at least 20 minutes. The professional light tapping method was adopted, that is, the index finger was fixed and the wrist was used to exert force. A draining/bouncing needle manipulation technique was employed, and both hands were used to grasp the affected area after puncture to squeeze out about $2 \mathrm{~mL}$ of blood. When the patient had registered the acupuncture sensation, C6805 electroacupuncture equipment (Shanghai Hanfei Medical Instrument Co., 
Ltd., Shanghai, China) was used with the continuous and dense wave modes at a frequency of $2 \mathrm{~Hz}$. The magnitude of stimulation was adjusted in real time according to each patient's level of tolerance. Whether the density of the treatment process was uniform or not was strictly monitored, and the volume of blood-letting was determined according to the tolerance of each individual.

On the basis of warming acupuncture and moxibustion at the temple, the research group was treated with Dailixin combined with drugs, which mainly included $0.5 \mathrm{mg}$ flurazide and $10 \mathrm{mg}$ melitracen hydrochloride. In this study, the drugs were obtained from Denmark Lundbeck Pharmaceutical Co., Ltd. (Copenhagen, Denmark). The study group was given 1 tablet at a time in the morning and afternoon following acupuncture treatment.

\section{Observation index}

Participants with TTH accompanied by anxiety or depression were treated in different ways in the two groups, the therapeutic effects on TTH in the two groups were compared, degree of headache before and after treatment was recorded, and the scores of the two groups were compared. In addition, the onset and duration of TTH were assessed and the related scores were compared between the two groups before and after treatment. In addition, the degree of pericardial muscle tenderness was compared between the two groups before and after treatment, and the Hamilton depression (HAMD) and Hamilton anxiety (HAMA) scores were recorded concurrently.

\section{Selection and screening criteria}

Participant inclusion criteria were as follows: (I) absence of mental health disorders or aphasia; ability to communicate with the researchers normally; (II) active stage of TTH was current at the time of recruitment/participation; (III) patients and their families understood the content of this study, provided written informed consent, and willingly volunteered to participate in this study; (IV) the condition of the selected patients met the diagnostic criteria of TTH stipulated by the International headache Association (IHS) and those of "middle head wind", which were judged by the "Diagnostic and Therapeutic Criteria of TCM Diseases and Syndromes" issued by the State Administration of Traditional Chinese Medicine (12).

The exclusion criteria were as follows: (I) compromised ability to express language and easily communicate with the researchers; (II) presence of mental health disorders or serious physical language impairment; (III) serious internal diseases such as abnormalities in visceral function of the heart, liver, kidney, or other vital organ; (IV) presence of any other factors that may have affected the results of this study; (V) all potential participants underwent neurological assessment and related computed tomography (CT) examination, patients with subsequently identified neurological diseases other than TTH were excluded, and patients with facial diseases such as glaucoma, diabetes, hypertension, anemia, brain trauma, and other internal and surgical diseases were also screened out of this study.

This study was approved by the Medical Ethics Committee of the Baotou Medical College (IRB-2016021) and was performed in accordance with Declaration of Helsinki (as revised in 2013).

\section{Statistical method}

The software SPSS version 20.0 (IBM Corp., Armonk, NY, USA) was used for statistical analysis of data. Measurement data were expressed as mean \pm standard deviation $\left(\bar{x}_{ \pm s}\right)$, and subjected to the $t$-test to determine significance. Count data were expressed as [n (\%)], and the test method was chisquare test. A $\mathrm{P}$ value $<0.05$ indicated statistical significance.

\section{Results}

\section{Comparison of therapeutic effects between the two groups}

Comparison of therapeutic effect between the two groups of patients revealed a significantly better treatment effect of the TTH in the study group than that of the control group $(\mathrm{P}<0.05)$. The difference between groups was statistically significant, in which the effective cure rate = clinical recovery + obvious effect + effective (Table 1).

\section{Comparison of headache score between the two groups before and after treatment}

The common clinical visual analogue score (VAS) was used to analyze the headache degree of participants: the 2 ends of a 10-cm long ruler were marked "painless" and "intense pain" respectively, and the participants marked the degree of pain according to their own experience. The scoring criteria were as follows: pain degree $\leq 2=1$ point; $>2$ and $\leq 4=2$ points; $>4$ and $\leq 6=3$ points; $>6$ and $\leq 8=4$ points; and $>8$ and $\leq 10=5$ points. The comparison of the scores between 
Table 1 Comparison of effective rates between the two groups

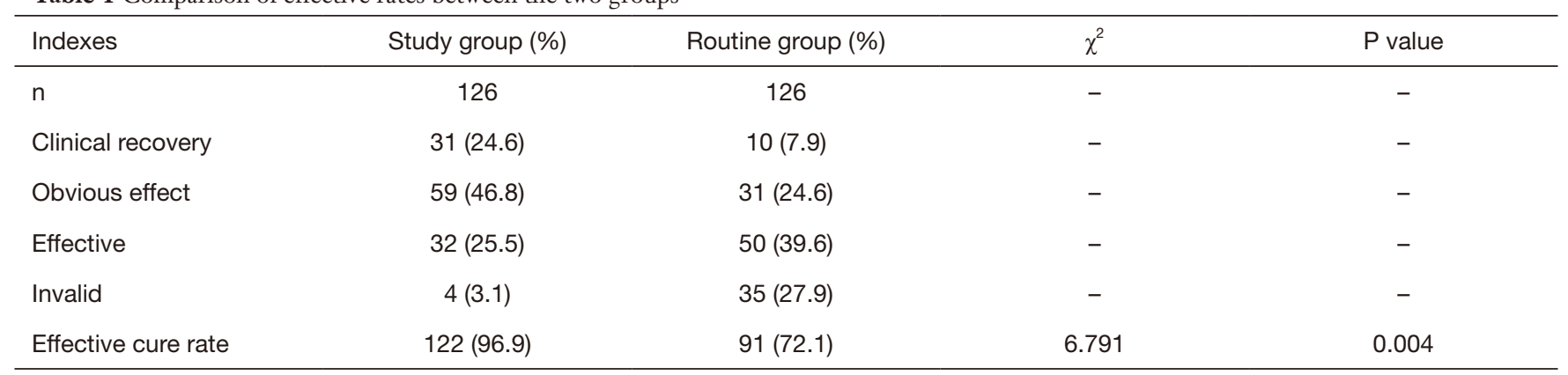

$\mathrm{P}<0.05$ indicates a significant statistical difference.

Table 2 Comparison of headache scores between the two groups before and after treatment $\left(\bar{x}_{ \pm}\right)$

\begin{tabular}{lccc}
\hline Groups & $\mathrm{n}$ & Before treatment & After treatment \\
\hline Study group & 126 & $2.69 \pm 0.22^{\#}$ & $1.21 \pm 0.12^{\star \#}$ \\
Routine group & 126 & $2.58 \pm 0.37$ & $2.21 \pm 0.20^{\star}$ \\
\hline
\end{tabular}

Compared with before treatment, ${ }^{*} \mathrm{P}<0.05$, there is statistical difference; compared with the routine group, ${ }^{\#} \mathrm{P}<0.05$, there is significant statistical difference.

Table 3 Comparison of the number and duration of headache attacks between the two groups before and after treatment $\left(\bar{x}_{ \pm s}\right)$

\begin{tabular}{lccccc}
\hline \multirow{2}{*}{ Groups } & \multicolumn{2}{c}{ Before treatment } & & \multicolumn{2}{c}{ After treatment } \\
\cline { 2 - 5 } \cline { 5 - 6 } & $\begin{array}{c}\text { Number of headache } \\
\text { attacks }\end{array}$ & $\begin{array}{c}\text { Duration of headache } \\
\text { attacks }\end{array}$ & & $\begin{array}{c}\text { Number of headache } \\
\text { attacks }\end{array}$ & $\begin{array}{c}\text { Duration of headache } \\
\text { attacks }\end{array}$ \\
\hline Study group $(\mathrm{n}=126)$ & $1.02 \pm 0.55^{\#}$ & $7.50 \pm 3.96^{\#}$ & $0.28 \pm 0.18^{* \#}$ & $0.59 \pm 0.02^{\star \#}$ \\
Routine group $(\mathrm{n}=126)$ & $1.05 \pm 0.50$ & $7.29 \pm 3.11$ & & $0.99 \pm 0.45^{*}$ & $1.01 \pm 0.39^{*}$ \\
\hline
\end{tabular}

Compared with before treatment, ${ }^{*} \mathrm{P}<0.05$, there is statistical difference; compared with the routine group, ${ }^{\#} \mathrm{P}<0.05$, there is significant statistical difference.

two groups before and after treatment showed that the headache degree score of the study group was significantly lower than that of the routine group $(\mathrm{P}<0.05)$ (Table 2$)$.

\section{Comparison of the frequency and duration of tension beadache between the two groups before and after treatment}

The number of headache attacks and the duration of each attack were recorded, which could be divided into 5 grades: 0 was no headache, 1 was mild headache, 2 was moderate headache, 3 was severe headache, and 4 was more severe headache. Headache duration was graded as follows: 1 for $\leq 2 \mathrm{~h}, 2$ for $2-6 \mathrm{~h}, 3$ for $6-24 \mathrm{~h}, 4$ for $24-72 \mathrm{~h}$, and 5 for $>72 \mathrm{~h}$. The number of attacks before and after treatment and their related durations were compared between the two groups of participants. The number and duration of headache attacks in the study group were significantly lower than those in the routine group $(\mathrm{P}<0.05)$, with significant statistical differences (Table 3).

\section{Comparison of pericranial muscle tenderness score between the two groups before and after treatment}

The degree of tenderness of pericranial muscle was compared between the two groups before and after treatment. Among them, 0 points indicated no tenderness upon muscle palpation; 1 point indicated mild tenderness on muscle palpation; 2 points indicated muscle tenderness upon palpation; and 3 points indicated obvious tenderness 
Table 4 Comparison of pericranial muscle tenderness scores between the two groups before and after treatment $\left(\bar{x}_{ \pm \mathrm{s}}\right)$

\begin{tabular}{lccc}
\hline Groups & $\mathrm{n}$ & Before treatment & After treatment \\
\hline Study group & 126 & $1.5 \pm 0.9^{\#}$ & $0.7 \pm 0.1^{\star *}$ \\
Routine group & 126 & $1.4 \pm 0.5$ & $1.2 \pm 0.8^{*}$ \\
\hline
\end{tabular}

Compared with before treatment, ${ }^{*} \mathrm{P}<0.05$, there is statistical difference; compared with the routine group, ${ }^{\#} \mathrm{P}<0.05$, there is significant statistical difference.

Table 5 Comparative table of HAMD and HAMA scores of the two groups before and after treatment $\left(\bar{x}_{ \pm \mathrm{s}}\right)$

\begin{tabular}{lcccc}
\hline Groups & \multicolumn{2}{c}{ Before treatment } & \multicolumn{2}{c}{ After treatment } \\
\cline { 2 - 4 } & HAMD & HAMA & HAMD & HAMA \\
\hline Study group $(n=126)$ & $15.19 \pm 3.56^{\#}$ & $13.21 \pm 4.49^{\#}$ & $2.01 \pm 1.51^{* \#}$ & $3.01 \pm 1.19^{* \#}$ \\
Routine group $(n=126)$ & $15.31 \pm 3.71$ & $13.18 \pm 4.71$ & $6.71 \pm 3.19^{*}$ & $8.29 \pm 3.99^{*}$
\end{tabular}

Compared with before treatment, ${ }^{*} P<0.05$, there is statistical difference; compared with the routine group, ${ }^{\#} \mathrm{P}<0.05$, there is significant statistical difference. HAMD, Hamilton depression score; HAMA, Hamilton anxiety score.

sensation on muscle palpation. The score of pericranial muscle tenderness in the study group was significantly lower than that in the routine group $(\mathrm{P}<0.05)($ Table 4$)$.

\section{Comparison of HAMD and HAMA scores between the two groups before and after treatment}

When the HAMD and HAMA scores of the two groups were compared after treatment, those of the study group were significantly lower than those of the routine group $(\mathrm{P}<0.05)$, and the difference was statistically significant (Table 5).

\section{Discussion}

Chronic tension-type headache belongs to primary headache, and is one of the most common types. The disease is often characterized by head pain and hoop sensation, rather than pulsatile pain. The course of the disease ranges from months to decades, and it is common in adults, especially in women. This kind of headache accounts for about $70 \%$ and $80 \%$ of headache patients. It is often caused by mental tension, too much thinking, too much pressure, and with the extension of the course of the disease, the aggravation of this kind of mental factors often lead to depression or anxiety in such patients. Therefore, most patients with chronic tension-type headache are often accompanied by anxiety or depression, and serious ones can affect daily life and work. The most common headache which presents in the clinical setting is TTH (13), which can be further divided into muscle contraction headache, stress headache, idiopathic headache, and psychogenic headache according to the cause of pain (14). The incidence of headache in China is high, as is the incidence of TTH (15). The purpose of this study was to analyze 252 typical headache patients treated in our hospital. These patients presented with some concurrent uncomfortable physical symptoms, such as gastrointestinal discomfort, lack of appetite, constipation or diarrhea, palpitation, sweating, limb pain, and so on. Tension-type headache, also known as muscle contraction headache, often due to mental tension, thinking too much, too much pressure and other accumulation time is too long, anxiety, depression or accompanied by insomnia, so that cerebral vascular irritant contraction, abnormal blood supply and lead to headache. A previous study found that the main cause of TTH was long-term neural activity. It is common for patients who experience intense mental stimulation to also have TTH $(16,17)$. The disorder of cerebral cortical activity caused by craniocerebral injury is another cause of TTH. When TTH is severe, insomnia occurs $(18,19)$, which can gradually evolve into depression, anxiety, and so on (20), thus forming a vicious cycle of mental stress and TTH. However, the etiology and pathogenesis of the disease are not clear $(21,22)$. The disease is mainly due to excessive mental stimulation and chronic improper posture of the neck $(23,24)$, which can lead to continuous contraction of the frontalis muscle, temporal muscle, cervical muscle, and scapular band muscle 
around the skull. As a result, the internal circulation of the muscle is hindered, resulting in decreased blood flow (25-27), head and neck pain, and in severe cases, it will promote the protective contraction of the head and neck muscles. Finally, a vicious circle is formed (28).

Patients with TTH can be treated with Deanxit, the effect of this drug is that with the continuous increase of medication time (29), the number of headache symptoms decrease and the physical symptoms gradually disappear. The patient's mental state and appetite improve, and the drug may have a certain effect on the prevention and recurrence of TTH (30). This study found that in the treatment of TTH, warming acupuncture combined with Deanxit is more effective than acupuncture alone, and the disease is mainly related to changes of dopamine, norepinephrine, and 5-hydroxytryptamine (31). Deanxit belongs to a compound preparation, which is mainly composed of haloperixol and melitracene (32). A small dose of Deanxit has an excitatory effect, and it combines with adrenaline, norepinephrine, serotonin, dopamine, and other neurotransmitters to elicit anti-anxiety, antidepressant, and mildly exhilarating effects, which will eventually improve the patient's physical symptoms, with obvious curative effect $(33,34)$. This study showed that warming acupuncture combined with Deanxit can significantly reduce the degree of headache, number and duration of headache attacks, and the related HAMD and HAMA scores of patients. At the same time, the treatment effect of the study group was significantly better than that of the routine group $(\mathrm{P}<0.05)$, and the difference was statistically significant.

To sum up, the application of warming acupuncture and moxibustion at the temple combined with Deanxit has achieved satisfactory results in the treatment of TTH with anxiety. Additionally, acupuncture treatment of TTH has the advantage of less side effects, and can improve muscle tissue ischemia and hypoxia. It has a significant effect on reducing the degree of headache in patients, which is worthy of extensive clinical promotion.

\section{Acknowledgments}

Funding: This study was supported by Natural Science Foundation of Inner Mongolia under grant (2019BS08002).

\section{Footnote}

Reporting Checklist: The authors have completed the
STROBE reporting checklist. Available at http://dx.doi. org/10.21037/apm-21-925

Data Sharing Statement: Available at http://dx.doi. org/10.21037/apm-21-925

Conflicts of Interest: All authors have completed the ICMJE uniform disclosure form (available at http://dx.doi. org/10.21037/apm-21-925). The authors have no conflicts of interest to declare.

Ethical Statement: The authors are accountable for all aspects of the work in ensuring that questions related to the accuracy or integrity of any part of the work are appropriately investigated and resolved. This study was approved by the Medical Ethics Committee of the Baotou Medical College (IRB-2016-021) and was performed in accordance with Declaration of Helsinki (as revised in 2013). Informed consent was taken from all the patients.

Open Access Statement: This is an Open Access article distributed in accordance with the Creative Commons Attribution-NonCommercial-NoDerivs 4.0 International License (CC BY-NC-ND 4.0), which permits the noncommercial replication and distribution of the article with the strict proviso that no changes or edits are made and the original work is properly cited (including links to both the formal publication through the relevant DOI and the license). See: https://creativecommons.org/licenses/by-nc-nd/4.0/.

\section{References}

1. Ertsey C, Magyar M, Gyüre T, et al. Tension type headache and its treatment possibilities. Ideggyogy $\mathrm{Sz}$ 2019;72:13-21.

2. Gildir S, Tüzün EH, Eroğlu G, et al. A randomized trial of trigger point dry needling versus sham needling for chronic tension-type headache. Medicine (Baltimore) 2019;98:e14520.

3. Shypilova EM, Zavadenko NN, Nesterovskiy YE. Preventive treatment of tension headache in children and adolescents. Zh Nevrol Psikhiatr Im S S Korsakova 2017;117:36-42.

4. Burch R, Rizzoli P, Loder E. The Prevalence and Impact of Migraine and Severe Headache in the United States: Figures and Trends From Government Health Studies. Headache 2018;58:496-505. 
5. Caamaño-Barrios LH, Galán-Del-Río F, Fernández-deLas-Peñas C, et al. Widespread Pressure Pain Sensitivity over Nerve Trunk Areas in Women with Frequent Episodic Tension-Type Headache as a Sign of Central Sensitization. Pain Med 2020;21:1408-14.

6. Skogvold L, Magnussen LH. Chronic tension-type headache and coping strategies in adolescents: a qualitative interview study. Physiother Res Int 2019;24:e1778.

7. Kim KM, Kim J, Cho SJ, et al. Excessive Daytime Sleepiness in Tension-Type Headache: A Population Study. Front Neurol 2019;10:1282.

8. Burch R. Migraine and tension-type headache: diagnosis and treatment. Med Clin North Am 2019;103:215-33.

9. Deodato M, Guolo F, Monticco A, et al. Osteopathic Manipulative Therapy in Patients With Chronic TensionType Headache: A Pilot Study. J Am Osteopath Assoc 2019. [Epub ahead of print]. doi: 10.7556/jaoa.2019.093.

10. Del Blanco Muñiz JA, Zaballos Laso A. Tension-type headache. Narrative review of physiotherapy treatment. An Sist Sanit Navar 2018;41:371-80.

11. Rizzoli P, Mullally WJ. Headache. Am J Med 2018;131:17-24.

12. Guo YW, Liang W, Peng MJ, et al. Acupuncture combined with Zhuang medicine thread moxibustion to treat tension headache case analysis. Asia-Pacific Traditional Medicine 2018;14:148-50.

13. Lee E, Moloney S, Talsma J, et al. Osteopathic Manipulative Treatment Considerations in Tension-Type Headache. J Am Osteopath Assoc 2019;119:e40-e41.

14. Spanou I, Bougea A, Liakakis G, et al. Relationship of Migraine and Tension-Type Headache With Hypothyroidism: A Literature Review. Headache 2019;59:1174-86.

15. Chassot M, Dussan-Sarria JA, Sehn FC, et al. Electroacupuncture analgesia is associated with increased serum brain-derived neurotrophic factor in chronic tension-type headache: a randomized, sham controlled, crossover trial. BMC Complement Altern Med 2015;15:144.

16. Benito-González E, Palacios-Ceña M, Fernández-Muñoz JJ, et al. Variables associated with sleep quality in chronic tension-type headache: A cross-sectional and longitudinal design. PLoS One 2018;13:e0197381.

17. Fernández-de-Las-Peñas C, Fernández-Muñoz JJ, Palacios-Ceña M, et al. Sleep disturbances in tensiontype headache and migraine. Ther Adv Neurol Disord 2017;11:1756285617745444.
18. Araki K. Diagnosis and treatment of primary headache (migraine and tension-type headache) in Japanese children and adolescents. No To Hattatsu 2012;44:119-24.

19. Doyle Strauss L, Weizenbaum E, Loder EW, et al. Amitriptyline Dose and Treatment Outcomes in Specialty Headache Practice: A Retrospective Cohort Study. Headache 2016;56:1626-34.

20. Hao XA, Xue CC, Dong L, et al. Factors associated with conflicting findings on acupuncture for tension-type headache: qualitative and quantitative analyses. J Altern Complement Med 2013;19:285-97.

21. McGeeney BE. Acupuncture is all placebo and here is why. Headache 2015;55:465-9.

22. Rizzoli P, Iuliano S, Weizenbaum E, et al. Headache in Patients With Pituitary Lesions: A Longitudinal Cohort Study. Neurosurgery 2016;78:316-23.

23. van Oosterhout WPJ, Cheung C, Haan J. Primary headache syndromes in the elderly: epidemiology, diagnosis and treatment. J Clin Transl Res 2016;2:45-51.

24. Lozano López C, Mesa Jiménez J, de la Hoz Aizpurúa $\mathrm{JL}$, et al. Efficacy of manual therapy in the treatment of tension-type headache. A systematic review from 20002013. Neurologia 2016;31:357-69.

25. Göbel H, Heinze A, Heinze-Kuhn K, et al. Peppermint oil in the acute treatment of tension-type headache. Schmerz 2016;30:295-310.

26. Do TP, Heldarskard GF, Kolding LT, et al. Myofascial trigger points in migraine and tension-type headache. J Headache Pain 2018;19:84.

27. Scripter C. Headache: Tension-Type Headache. FP Essent 2018;473:17-20.

28. Cheraghi F, Shamsaei F, Fayyazi A, et al. Comparison of the Quality of Sleep and Intensity of Headache between Migraine, Tension Headache, and Healthy Children. Iran J Child Neurol 2018;12:45-54.

29. Eidlitz-Markus T, Zolden S, Haimi-Cohen Y, et al. Comparison of comorbidities of migraine and tension headache in a pediatric headache clinic. Cephalalgia 2017;37:1135-44.

30. de Tommaso M, Fernández-de-Las-Penas C. Tension Type Headache. Curr Rheumatol Rev 2016;12:127-39.

31. Linde K, Allais G, Brinkhaus B, et al. Acupuncture for the prevention of tension-type headache. Cochrane Database Syst Rev 2016;4:CD007587.

32. Joshi S, Rizzoli P, Loder E. The comorbidity burden of patients with cluster headache: a population-based study. J Headache Pain 2017;18:76. 
33. Caballero-Calixto L, Puescas A, Reyna-Ormeño J. The effect of manual therapy on tension headache. Neurologia 2017;32:201-2.

34. May A. Hints on Diagnosing and Treating Headache.

Cite this article as: A R, Bao Q, Sai YC, Te M, Hu R, Sa R, $\mathrm{Mu}$ R, Bo A. Clinical observation of warming acupuncture and moxibustion at the temples combined with Deanxit in the treatment of tension headache with anxiety and depression: a retrospective study. Ann Palliat Med 2021;10(5):5541-5548. doi: 10.21037/apm-21-925
Dtsch Arztebl Int 2018;115:299-308.

(English Language Editor: J. Jones) 